\title{
Oedema reduction by mechanical compression improved the healing of foot infection in patients with diabetes mellitus
}

\author{
Armstrong DG, Nguyen HC. Improvement in healing with aggressive edema reduction after debridement of foot infection \\ in persons with diabetes. Arch Surg 2000 Dec;135:1405-9.

\section{QUESTION: Does aggressive oedema reduction with a foot level mechanical compression device improve healing of foot infection in patients with diabetes mellitus?}

\section{Design}

Randomised \{allocation concealed*\} $\uparrow$, blinded (investigators and patients),* controlled trial with 12 weeks follow up.

\section{Setting}

University teaching hospital and related clinics in San Antonio, Texas, USA.

\section{Patients}

115 patients with diabetes who had foot infections requiring incision and debridement. Exclusion criteria were congestive heart failure, advanced renal disease, serum creatinine concentration $>177 \mu \mathrm{mol} / \mathrm{l}$, or planned lower extremity bypass graft surgery. 97 patients (84\%) (mean age 50 y, $74 \%$ men) had complete follow up.

\section{Intervention}

Patients were allocated to receive a functioning (active group, $\mathrm{n}=59$ ) or non-functioning (placebo group, $\mathrm{n}=56$ ) pulsatile pneumatic foot compression system. This system included a bladder containing foot wrap and a pump that intermittently fired bursts of air through tubing to the wrap, thereby emptying the foot veins. Patients were asked to use the device 8 hours/day. In addition, patients had weekly wound care that included debridement of the site, and they were given wound care instructions.

Source of funding: Kinetic Concepts Inc, San Antomio, Texas.

For correspondence: Dr D G Armstrong, Department of Surgery, Southern Arizona Veterans Affairs Medical Center, 3601 South Sixth Avenue, Tucson, $A Z 85723$ USA.Fax +1781823 6628

\section{Main outcome measures}

Oedema reduction and rate of complete foot wound healing.

\section{Main results}

Patients in the active group had a higher rate of complete foot wound healing $(\mathrm{p}<0.02)$ (table) and a greater degree of oedema reduction as shown by mean foot circumference measurement $(23.8 v 25.7 \mathrm{~cm}$, $\mathrm{p}<0.001)$ than those in the placebo group.

\section{Conclusion}

A foot level mechanical compression device reduced oedema and improved healing of foot ulcers in patients with diabetes mellitus.

*See glossary.

$\dagger$ Information provided by author.

\section{COMMENTARY}

Foot complications in people with diabetes are an important cause of morbidity and mortality. They are expensive to treat and often require admission to hospital for surgery and rehabilitation; therefore, preventing them should be a priority. ${ }^{1}$ Few good quality trials exist on the treatment of foot ulceration in diabetes. ${ }^{2}$ Current best practice emphasises regular and thorough wound debridement and avoidance of weight bearing activities. Armstrong and Nguyen used these principles in all their patients, and their study adds to our understanding of the pathogenesis of foot ulcers by showing that less oedema is associated with better healing.

This study raises some minor concerns. Although the mean baseline wound size did not differ between the groups, the mean and standard deviation were both smaller in the actively treated patients, which might suggest a baseline imbalance in the numbers of large ulcers. More ulcers affected the mid or hind foot in the placebo group (38\% v $29 \%$ ). $16 \%$ of patients dropped out of the study for various reasons, and a substantial number in both groups (numbers unspecified) were unable to tolerate the device for 8 hours/ day. The proportions of ischaemic and neuropathic ulcers were also not reported.

None the less, this carefully done randomised controlled trial is welcome. Its hypothesis is well founded, and the results are promising if not quite conclusive. A further study with randomisation by ulcer size, location, and cause is needed before regular use of these devices can be recommended. Meanwhile, the impressive healing rate of $>50 \%$ in the placebo group by using expert debridement underlines the importance of this treatment for all our patients.

Rudy W Bilous, MD Middlesbrough General Hospital Middlesbrough, Cleveland, UK

1 Mason J, O'Keeffe C, McIntosh A, et al. A systematic review of foot ulcer in patients with type 2 diabetes mellitus. I: prevention. Diabet Med 1999;16:801-12.

2 Mason J, O'Keeffe C, Hutchinson A, et al. A systematic review of foot ulcer in patients with type 2 diabetes mellitus. II: treatment. Diabet Med 1999;16:889-909. 